\title{
Doppler Parameters in Renal Transplant Dysfunction
}

Correlations With Histopathologic Changes

\author{
Jing Gao, MD, Jonathan M. Rubin, MD, PhD, Dong-ying Xiang, MD, Wen He, MD, Yong Ho Auh, MD, \\ John Wang, MD, Amelia Ng, RN, Robert Min, MD
}

Article includes CME test

Received July 13, 2010, from the Department of Radiology (J.G., Y.H.A., A.N., R.M.) and Rogosin Institute and Division of Nephrology (J.W.), New York-Presbyterian Hospital, Weill Cornell Medical Center, New York, New York USA; Department of Radiology, University of Michigan Hospitals, Ann Arbor, Michigan USA (J.M.R.); and Department of Ultrasound, Tiantan Hospital, Capital Medical University, Beijing, China (D.X., W.H.). Revision requested August 30, 2010. Revised manuscript accepted for publication September 22, 2010.

Address correspondence to Jing Gao, $M D$, Department of Radiology, New York-Presbyterian Hospital, Weill Cornell Medical Center, 528 E 68th St, Suite 8A-27, New York, NY 10065 USA.

E-mail:jig2001@med.cornell.edu

Abbreviations

EDV, end-diastolic velocity; PSV, peak systolic velocity; RI, resistive index
Objectives - The aim of this study was to assess the relationship between intrarenal Doppler parameters and histopathologic changes shown on kidney biopsy in renal transplant dysfunction.

Methods-We retrospectively reviewed the records of 113 patients (61 men and 52 women; age range, 22-76 years; mean age \pm SD, $50.9 \pm 12.7$ years) who underwent both transplanted kidney sonography and biopsy from May 1, 2007, to May 31, 2009. Doppler parameters of the interlobar arteries, including the peak systolic velocity (PSV), end-diastolic velocity (EDV), and resistive index (RI), were compared with kidney biopsy findings. According to histopathologic findings, the 113 patients were divided into two groups: 1, interstitial fibrosis/tubular atrophy and vascular/glomerular sclerosis $(n=79)$; and 2, edematous changes in glomeruli without fibrosis $(n=34)$. The correlations between Doppler parameters and histopathologic findings were statistically analyzed.

Results - There were statistically significant differences in the PSV and EDV of the interlobar arteries between groups 1 and 2 . Both the PSV and EDV in group 1 were significantly lower than those in group $2(P<.001)$. There was no significant difference in the $\mathrm{RI}$ of the interlobar arteries between the two groups $(P>.05)$. There were no significant differences in the PSV, EDV, and RI of the main renal artery between the two groups (all $P>.05)$.

Conclusions - The PSV and EDV of the interlobar artery have statistical correlations with histopathologic types in renal transplant dysfunction. Both the PSV and EDV in interstitial fibrosis/tubular atrophy and vascular/glomerular sclerosis seem lower than those in glomerulopathy without fibrosis. Hence, the PSV and EDV of the interlobar artery may potentially be used as hemodynamic indicators for monitoring the progress of renal transplants.

Key Words_-Doppler parameters; histopathologic changes; renal transplant dysfunction

K idney biopsy is considered an essential tool that provides histopathologic information about an impaired renal transplant. However, biopsy is invasive and may be contraindicated in patients with certain disorders such as coagulopathy. Moreover, kidney biopsy complications, such as intrarenal arteriovenous fistulas with a "steal" and large perinephric hematomas, may eventually lead to or worsen renal failure. ${ }^{1}$ Alternatively, sonography, an established noninvasive imaging study, has become a routine application for evaluating renal transplants. 
Morphologic changes, including the size, parenchymal echogenicity, and corticomedullary differentiation, of the transplanted kidney on gray scale imaging may occur in a graft with dysfunction. Unfortunately, these sonographic appearances in the evaluation of renal failure lack specificity. ${ }^{2}$ Moreover, it has been reported that renal morphologic alterations appear much later than biochemical changes, such as a rising serum creatinine level, developing after existing impaired circulation in intrarenal vessels. ${ }^{3}$

The resistive index (RI) has been used as an indicator for evaluating the hemodynamics of transplanted and native kidneys. ${ }^{4,5}$ Some publications have found the RI to be a valuable Doppler parameter in the assessment of renal transplant dysfunction, ${ }^{6-11}$ whereas other studies have been inconclusive. ${ }^{12-17}$ Finally, a number of researchers have agreed that comparison of follow-up and initial RI values may provide useful information for monitoring the progress of the allograft, evaluating therapeutic efficacy, and detecting subclinical atherosclerotic damage in the cardiovascular system of transplant recipients. ${ }^{10,12}$

A review of the literature found no reports on the correlation between decreased Doppler velocity in the interlobar arteries and histopathologic changes in an impaired renal transplant. The aim of our study was to assess the correlation of alterations in Doppler parameters, not only the $\mathrm{RI}$ but also the peak systolic velocity (PSV) and enddiastolic velocity (EDV), with histopathologic changes in renal transplant dysfunction. The ultimate goal would be to show the use of the PSV and EDV as hemodynamic predictors or indicators for monitoring the progress of renal transplants.

\section{Materials and Methods}

\section{Patient Information}

We retrospectively reviewed the records of 492 patients who had renal transplant sonography from May 1, 2007, to May 31, 2009. No consent form was needed because this was a retrospective report. The study was approved by the Institutional Review Board of Weill Cornell Medical College (approval number 0812010120) and was Health Insurance Portability and Accountability Act compliant.

Of the 492 patients, 113 (61 men and 52 women; age range, $22-76$ years; mean age $\pm S D, 50.9 \pm 12.7$ years) had renal transplant dysfunction (creatinine level, $>1.1$ $\mathrm{mg} / \mathrm{dL}$ ) and underwent both biopsy and sonography from May 1, 2007, to May 31, 2009. Nine patients had living donors ( $\mathrm{n}=5$ in group 1 and $\mathrm{n}=4$ in group 2 ), and $104 \mathrm{had}$ cadaveric donors $(\mathrm{n}=74$ in group 1 and $\mathrm{n}=30$ in group 2$)$. The time frame for performing sonography after trans- plantation ranged between 1 day and 19.9 years (mean, 1.6 years). According to the Banff 07 updated classification for renal transplant allograft biopsy pathologic criteria, ${ }^{18}$ the patients were divided into two groups. Group $1(n=79)$ had interstitial fibrosis/tubular atrophy (grades 5.I, 5.II, and 5.III) in the tubulointerstitial compartment and vascular/glomerular sclerosis caused by rejection or tubular necrosis and medication toxicity. Group $2(\mathrm{n}=34)$ had edema and inflammation in glomeruli without fibrosis, resulting from acute rejection, or glomerulonephritis. The interval between renal transplant sonography and kidney biopsy was less than 3 days. Renal transplant sonography was requested by the nephrologist and transplant surgeon at our institution.

Patients with congestive heart failure, major stenosis of the aorta, iliac artery, or transplanted kidney, an existing intrarenal arteriovenous fistula, a large perinephric collection, hydronephrosis, and hypertension were excluded from this study because these conditions may affect the intrarenal blood flow velocity during systole and diastole. Patients who had a duration of more than 3 days between renal transplant sonography and kidney biopsy were also excluded.

\section{Color and Spectral Doppler Sonography}

Color Doppler sonography was performed with 2- to 4$\mathrm{MHz}$ curved linear array or phased array sector transducer (Sequoia 512, Siemens Medical Solutions, Mountain View, CA; and LOGIQ9, GE Healthcare, Milwaukee, WI). There was no special preparation before renal transplant sonography.

Patients were scanned in a supine or decubitus position to achieve an ultrasound beam as nearly parallel to the blood flow direction at the intrarenal artery as possible. An ultrasound probe covered with transmitting gel was gently placed on the skin over the transplanted kidney. Minimal pressure was applied during the scanning to avoid mechanical compression on the kidney, which can cause a false-positive elevation of the RI. ${ }^{10} \mathrm{~A}$ low pulse repetition frequency and filter were used to detect the low-velocity flow. Color flow imaging was used to locate the interlobar artery at the junction of the medulla and pyramids (Figure 1 ). Doppler angle correction of less than $60^{\circ}$ (the best Doppler angle would with the sound beam parallel to the direction of flow at the interlobar artery), a 2- to 4-mm spectral Doppler gate, and a low scale without aliasing were standard for sampling blood flow velocity with spectral Doppler. The PSV was routinely measured in the iliac artery, arterial anastomosis of the renal and iliac arteries, and main transplant renal artery for arterial stenosis screening. ${ }^{1}$ The EDV and RI were also measured in the main trans- 
plant renal artery. Although the interlobular artery is closer to the glomerulus than the interlobar artery, with most currently used commercial ultrasound scanners, the interlobular artery is, unfortunately, too small to optimally visualize. Therefore, interlobar arteries are the vessels of choice when measuring blood flow in the kidney with spectral Doppler analysis. 19,20

The angle-corrected PSV (highest velocity at systole), EDV (minimal diastolic velocity), and RI [(PSV $\mathrm{EDV}) / \mathrm{PSV}]$ on the spectra were manually measured with built-in software in the ultrasound scanner (Figure 2). The Doppler parameters measured from the upper, mid, and lower poles of the transplanted kidney were averaged, which was defined as $P S V_{\text {mean }}=\left(\mathrm{PSV}_{\text {upper pole }}+\mathrm{PSV}_{\text {mid pole }}\right.$ $\left.+\mathrm{PSV}_{\text {lower pole }}\right) / 3$. Subsequently, the mean PSV, EDV, and RI were calculated for each patient.

\section{Histopathologic Examination}

Percutaneous kidney biopsies were performed with an 18gauge biopsy needle (Bard Peripheral Technologies, Covington, GA) under sonographic localization at the lower pole of the transplanted kidneys. All biopsies were performed after renal transplant sonography to exclude potential intrarenal arteriovenous fistulas and pseudoaneurysms. The kidney biopsies were done in the ultrasound room of the Department of Radiology. After kidney biopsy, the specimens were sent to the Department of Pathology. Biopsy specimens were reviewed by an experienced pathologist who had worked on renal histopathologic diagnosis in native and transplanted kidneys for 20 years. The pathologist never reviewed the sonograms or sonographic findings before interpreting the pathologic specimens, so the

Figure 1. Longitudinal color flow image of a transplanted kidney showing the interlobar artery (arrow) at the junction of the pyramids and medulla. The flow direction in the interlobar artery is toward the transducer (red).

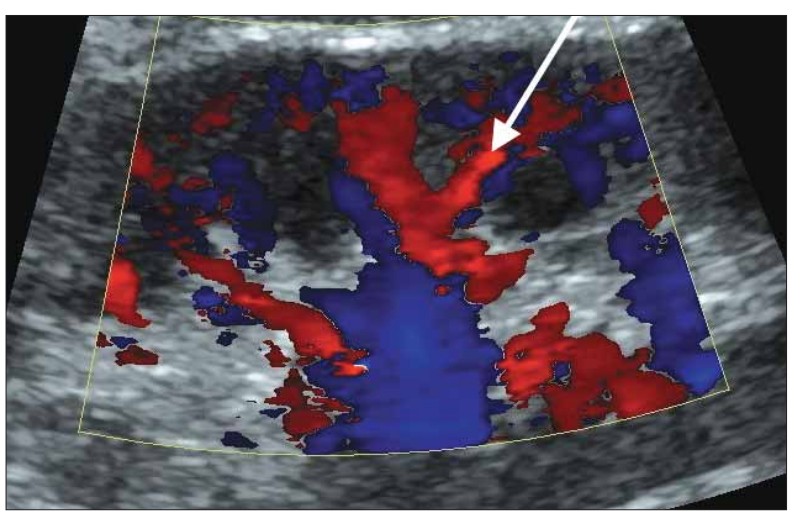

histopathologic reviews were essentially blinded regarding the results of renal transplant sonography. The site of the pathologic change and dominant process (fibrosis, atrophy, edema, or arteriosclerosis) were classified depending on the findings primarily located in the interstitium, tubules, vessels, or glomeruli according to the updated Banff 07 renal allograft pathologic classification. ${ }^{18}$

\section{Statistical Analysis}

SPSS version 16.0 software (SPSS Inc, Chicago, IL) was used for statistical analysis. All of the Doppler parameters were expressed as mean \pm SD. An unpaired Student $t$ test was used to statistically analyze different PSV, EDV, and $\mathrm{RI}$ values in the main renal artery and interlobar artery as well as patient ages between groups 1 and 2 . An independent $t$ test was used to test the differences between the transplantation durations between the two groups after logarithmically transforming a non-normal distribution of the transplantation durations into normal distribution data. $P<.05$ was defined as statistically significant.

\section{Results}

On the basis of the histopathologic findings, 79 patients were included in group 1, with interstitial fibrosis/tubular atrophy (grades 5.I, 5.II, and 5.III) and vascular/glomerular sclerosis resulting from rejection, tubular necrosis, and

Figure 2. Color duplex sonogram from a patient with renal transplant dysfunction (serum creatinine level, $3.2 \mathrm{mg} / \mathrm{dL}$ ) 227 weeks after transplantation. The Doppler spectrum is sampled at the interlobar artery of the transplanted kidney on a zoomed longitudinal color duplex image. The spectral Doppler angle of $5^{\circ}$ is nearly parallel to the flow direction in the interlobar artery (arrow). The Doppler gate is $3.0 \mathrm{~mm}$. The peak systolic velocity (V1), end-diastolic velocity (V2), and resistive index (RI) are $0.189 \mathrm{~m} / \mathrm{s}(18.9 \mathrm{~cm} / \mathrm{s}), 0.050 \mathrm{~m} / \mathrm{s}(5.0 \mathrm{~cm} / \mathrm{s})$, and 0.73 , respectively. S/D indicates systolic to diastolic ratio. Interstitial fibrosis and tubular atrophy were seen in $50 \%$ of the allograft on histologic examination (not shown).

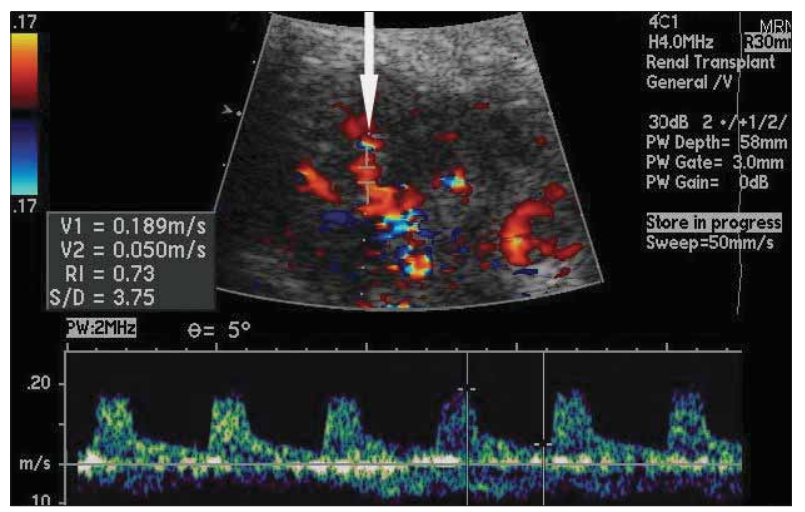


medication toxicity. Thirty-four patients were included in group 2, with edema without fibrosis in glomeruli caused by acute rejection, inflammation, and diabetic or lupus glomerulopathy as the major histopathologic finding.

For the main renal artery, the PSV, EDV, and RI were $98.96 \pm 8.60 \mathrm{~cm} / \mathrm{s}, 20.75 \pm 4.42 \mathrm{~cm} / \mathrm{s}$, and $0.77 \pm 0.05$, respectively, in group 1 and $101.71 \pm 11.77 \mathrm{~cm} / \mathrm{s}, 21.47 \pm$ $4.41 \mathrm{~cm} / \mathrm{s}, 0.78 \pm 0.05$, in group 2 (Table 1). There were no statistically significant differences in the PSV, EDV, and $\mathrm{RI}$ of the main renal artery between the two groups (all $P<.05)$. For the interlobar artery, the PSV was $23.11 \pm$ $7.37 \mathrm{~cm} / \mathrm{s}$ in group 1 , which was significantly lower than $39.05 \pm 11.22 \mathrm{~cm} / \mathrm{s}$ in group $2(P<.001)$. The EDV in group 1 was also significantly lower than in group 2 ( 6.68 \pm 3.31 versus $10.32 \pm 4.14 \mathrm{~cm} / \mathrm{s} ; P<.001)$. There was no statistically significant difference in the RI between groups 1 and $2(0.71 \pm 0.12$ versus $0.73 \pm 0.09 ; P>.05$; Table 1 and Figure 3$)$. There were no significant differences in ages and transplantation durations between the two groups $(P>.05$; Table 2$)$.

\section{Discussion}

Serum creatinine screening and sonography are commonly used to assess the function and condition of renal transplants. However, it takes time for the creatinine level to rise and for alterations in the morphologic and mechanical properties of the allograft to occur after the inception of circulation impairment in the interlobar artery, where the blood flow velocity is the most effective hemodynamic in the glomerular filtration rate of a renal transplant. ${ }^{19,20}$ Therefore, the renal vasculature on color flow imaging and blood flow velocity on spectral Doppler imaging are routinely assessed during renal transplant sonography. Despite remarkable improvements in the sensitivity of evaluating low-velocity flow in renal transplants with power and color Doppler imaging, spectral Doppler imaging has changed little over the last 2 decades. Clinical quantification of the blood flow velocity near the glomerulus with a noninvasive imaging method still relies on Doppler measurements in the interlobar artery, including the PSV and EDV. Moreover, the hemodynamic state, eg, renal vascular resistance, is assessed by the $\mathrm{RI},{ }^{21}$ although the role of the RI in evaluation of renal failure remains controversial. Recent studies on the ultrasound elasticity of renal transplant allografts have provided a new method that assesses the stiffness of the renal parenchyma in chronic renal transplant dysfunction. ${ }^{22}$ Harder (ie, stiffer) renal parenchyma associated with interstitial fibrosis may gradually develop secondarily after a long period of insufficient renal hemodynamics.

It has been hoped that a noninvasive technique could be used to detect and monitor the beginning or early stages of renal hemodynamic disturbances that may lead to renal transplant dysfunction. Therefore, multiple investigations on Doppler parameters have been conducted, with most studying the RI. ${ }^{2-33}$ As a result, there have been encouraging reports on the $\mathrm{RI}$ as a valuable tool for monitoring renal conditions from some institutions ${ }^{6-11}$ as well as disappointing conclusions about using the RI for assessing renal diseases from others. ${ }^{12-17}$

One study suggested that the site of renal histopathologic changes was more important than their severity in producing an elevated RI. ${ }^{33}$ This was in accordance with another report, which found that active or acute diseases within the tubulointerstitial compartment and vasculitis generally caused an elevated RI, whereas diseases limited primarily to the glomeruli did not. ${ }^{4}$ In our study, there was no marked difference in the RI for interstitial fibrosis/tubular atrophy in the tubulointerstitial compartment versus edema in the glomeruli without fibrosis in impaired renal transplants $(P>.05$; Table 1$)$. To find out how the RI failed to live up to its promise as a parameter for measuring changes in renal transplants, we reviewed the theoretical explanations for understanding the meaning, usefulness, and limitations of the RI. ${ }^{4,5,21,23-26}$

Table 1. Doppler Parameters and Clinical Information for 113 Patients With Renal Transplant Dysfunction

\begin{tabular}{|c|c|c|c|c|}
\hline Parameter & Group $1(n=79)$ & Group $2(n=34)$ & $t$ Test & $P$ \\
\hline Interlobar artery PSV, cm/s & $23.11 \pm 7.37$ & $39.05 \pm 11.22$ & -8.932 & $<.001$ \\
\hline Interlobar artery EDV, cm/s & $6.68 \pm 3.31$ & $10.32 \pm 4.14$ & -4.932 & $<.001$ \\
\hline Interlobar artery RI & $0.71 \pm 0.12$ & $0.73 \pm 0.09$ & -0.668 & $>.05$ \\
\hline Main renal artery PSV, $\mathrm{cm} / \mathrm{s}$ & $98.96 \pm 8.60$ & $101.71 \pm 11.77$ & 1.679 & $>.05$ \\
\hline Main renal artery EDV, $\mathrm{cm} / \mathrm{s}$ & $20.75 \pm 4.42$ & $21.47 \pm 4.41$ & 0.799 & $>.05$ \\
\hline Main renal artery RI & $0.77 \pm 0.05$ & $0.78 \pm 0.05$ & 0.313 & $>.05$ \\
\hline
\end{tabular}

Quantitative variables are expressed as mean \pm SD. Group 1 includes patients with interstitial fibrosis, tubular atrophy, and vascular/glomerular sclerosis; group 2 includes patients with edematous changes in glomeruli without fibrosis. EDV indicates end-diastolic velocity; PSV, peak systolic velocity; and RI, resistive index. 


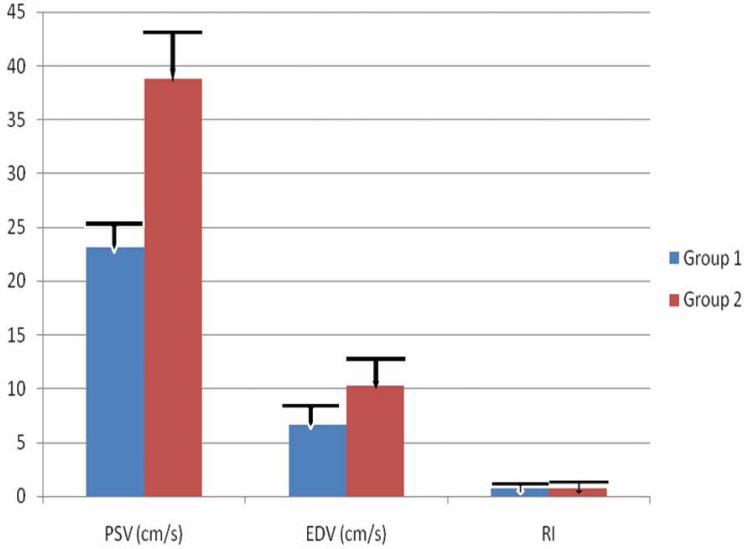

Figure 3. Distribution of Doppler parameters, including the peak systolic velocity (PSV), end-diastolic velocity (EDV), and resistive index (RI), in 113 cases of renal transplant dysfunction. The PSV and EDV in the renal transplant allografts with interstitial fibrosis/tubular atrophy and arteriosclerosis in group 1 ( $n=79$; left bars) are significantly lower than in those with glomerulopathy without interstitial fibrosis/tubular atrophy in group 2 ( $n=34$; right bars). However, there is no significant difference in the RI between the two groups.

The RI is the ratio of the difference between the PSV and EDV to the PSV. Theoretically, a nonproportional alteration of either the PSV or EDV or both may change the RI. Clinically, varying appearances of the Doppler waveform and velocity in the interlobar artery are due to interactions between the many renal hemodynamic factors that are produced, which are sometimes altered in response to changes caused by extrarenal (prerenal and postrenal) conditions, intrarenal conditions, or both. Extrarenal condi- tions that may affect renal blood flow and substantially alter the Doppler waveform include the cardiac output, blood pressure, right-sided heart function, donor's age, and vascular condition of the recipient. The main intrarenal conditions that may cause hemodynamic changes are glomerulopathy, interstitial fibrosis, tubular atrophy, and arteriosclerosis. ${ }^{28-}$ ${ }^{31}$ Coexisting factors are not unusual in renal transplants, and combinations of several pathologic changes in the tubulointerstitial compartment and glomeruli are quite common, which can make interpretation of Doppler parameters in an impaired renal transplant difficult.

The PSV and EDV are semiquantitative measures of intrarenal blood flow on spectral Doppler imaging, and they strongly depend on the distension of small arteries in the kidney. Thus, the PSV and EDV are associated with renal vascular compliance and vascular resistance. ${ }^{10,21,23-}$ ${ }^{26}$ Murphy and Tublin ${ }^{24}$ described an elevated renal interstitial pressure that would counteract the distension of arteries and arterioles. Because this counteraction would not be identical during systole and diastole, the elevated interstitial pressure would have asymmetric effects on systolic and diastolic blood flow as well as blood velocity. This effect is more dramatic during diastole. ${ }^{24}$ Bude and Rubin ${ }^{25}$ also reported that the diastolic velocity decreased after the reduction of compliance, and it could be immeasurable at zero compliance. Our results suggest that decreased renal vascular distension further reduces the effect on intrarenal blood flow in a cross section of renal vessels during both systole and diastole (Figure 3), particularly its effect on the diastolic velocity. The PSV and EDV changes in the interlobar artery may have resulted from either poor vascular compliance due to interstitial fibrosis, which limited dis-

Table 2. Demographic Information for 113 Patients With Renal Transplant Dysfunction

\begin{tabular}{|c|c|c|c|}
\hline Characteristic & Group $1(n=79)$ & Group $2(n=34)$ & $P$ \\
\hline Male/female & $41 / 38$ & 20/14 & NA \\
\hline \multirow[t]{2}{*}{ Donor } & Living, 5 & Living, 4 & NA \\
\hline & Cadaveric, 74 & Cadaveric, 30 & \\
\hline Age, y & $49 \pm 2.8$ & $53 \pm 1.9$ & $>.05$ \\
\hline Transplantation duration, y & $1.9 \pm 2.8$ & $0.9 \pm 2.0$ & $>.05$ \\
\hline \multirow[t]{3}{*}{ Race } & African American, 17 & African American, 6 & NA \\
\hline & Asian, 9 & Asian, 5 & \\
\hline & White, 53 & White, 23 & \\
\hline \multirow[t]{6}{*}{ Underlying etiology of renal failure } & Vascular/cellular rejection, 31 & Recurrent glomerulonephritis, 8 & NA \\
\hline & Tubular necrosis/atrophy, 23 & Lupus nephropathy, 6 & \\
\hline & Renal vessel thrombosis, 3 & Diabetic nephropathy, 13 & \\
\hline & Focal coagulative necrosis, 2 & Pyelonephritis, 5 & \\
\hline & Arterio-arteriolosclerosis, 11 & Peritubular capillary inflammation, 2 & \\
\hline & Calcineurin inhibitor toxicity, 9 & & \\
\hline
\end{tabular}

Quantitative variables are expressed as mean \pm SD. Group 1 includes patients with interstitial fibrosis, tubular atrophy, and vascular/glomerular sclerosis; group 2 includes patients with edematous changes in glomeruli without fibrosis. NA indicates not applicable. 
tension of the artery, or stiffness of the artery itself from arteriosclerosis $^{3}$ in group 1 or from increased interstitial pressure caused by glomerular edema ${ }^{13}$ in group 2 . All statistical results in our study indicate that the blood flow changes in the interlobar artery during both systole and diastole are closely associated with a certain renal histopathologic type. The effect of fibrosis in the tubulointerstitium on decreasing vascular distension was more profound than the effect of edema in glomeruli without fibrosis. This affected blood flow velocities during both diastole and systole in the interlobar artery, whereas in our observation, it did not in the main renal artery (Table 1). Asphyxia could cause a decrease in blood flow in the interlobar artery, manifested as a low EDV, the Doppler parameter that had most significant negative correlation with the severity of asphyxia, which may cause edematous changes in the glomeruli and interstitial compartment. ${ }^{27}$ The EDV may be decreased in early transplantation grafts with acute rejection and acute tubular necrosis due to ischemic edema, whereas interstitial fibrosis/tubular atrophy may not yet have developed.

When using Doppler sonography to assess renal transplant hemodynamics, the PSV and EDV in the interlobar artery should be taken into consideration when interpreting Doppler parameters and analyzing the correlations between these measurements and clinical data or biochemical results. In our study, there was no significant difference in the RI between the two groups; however, the PSV in group 1 was significantly lower than in group $2(P<$ $.001)$, and the EDV in group 1 was also markedly lower than in group $2(P<.001)$ in the interlobar arteries. The explanation for these statistical results would be that the decreases of both the PSV and EDV in group 1 were relatively proportional to those in group 2. Although there were significant differences in the PSV and EDV between the two groups, the transplantation durations between the two groups were not significantly different $(P>.05)$. This may have resulted from the use of marginal donors, the existence of arteriosclerosis in the recipients, and transplantation from living related donors. The time for detecting interstitial fibrosis/tubular atrophy and arteriosclerosis in renal transplant allografts by kidney biopsy is not absolutely associated with the transplantation duration; for instance, interstitial fibrosis/tubular atrophy could be detected in a marginally hypertensive donor during the first week of transplantation because the fibrosis and arteriosclerosis could have developed before the transplantation. It could also be seen in a living related donor several years after transplantation if no rejection or other complications occur before renal transplant biopsy. Therefore, the range of transplantation durations in our study was large.
With regard to Doppler parameters in the intrarenal artery, our study does not suggest that any Doppler parameter can replace kidney biopsy in managing renal transplants. We intended to assess the correlation between Doppler parameter alterations and certain renal histopathologic types such as interstitial fibrosis/tubular atrophy and glomerulopathy.

The limitations of our study included interobserver variation in ultrasound scanning and a non-normal distribution of the transplantation durations. Other limitations included a lack of standardization regarding the times kidney biopsy and renal transplant sonography were performed, allograft types (living versus cadaveric donors), and underlying etiology of renal failure, such as lupus nephropathy and diabetic nephropathy.

In conclusion, it seems that the theory and interpretation of Doppler parameters in renal diseases are more complex than originally thought. High resistance and poor compliance in renal vessels would decrease renal vascular distensibility and the cross section of renal vessels, which may consequently result in decreasing intrarenal blood flow during systole, diastole, or both. Hence, the PSV and EDV in the interlobar artery may potentially be used as indicators for detecting hemodynamic insufficiency that may result in renal transplant dysfunction. More research on Doppler parameters that have potential implications for noninvasive investigation of kidney transplant hemodynamics should be encouraged.

\section{References}

1. Gao J, NgA, Shih G, et al. Intrarenal color duplex ultrasonography: a window to vascular complications of renal transplants. J Ultrasound Med 2007; 26:1403-1418.

2. Park SB, Kim JK, Cho KS. Complications of renal transplantation: ultrasonographic evaluation. JUltrasound Med 2007; 26:615-633.

3. Langer JE, Jones LP. Sonographic evaluation of the renal transplant. Ultrasound Clin 2007; 2:73-88.

4. PlattJF, Rubin JM, Ellis JH. Lupus nephritis: predictive value of conventional and Doppler US and comparison with serologic and biopsy parameters. Radiology 1997; 203:82-86.

5. PlattJF. Doppler ultrasound of the kidney. Semin Ultrasound CT MR 1997; 18:22-32

6. Khosroshahi HT, Tarzamni M, Oskuii RA. Doppler ultrasonography before and 6 to 12 months after kidney transplantation. Transplant Proc 2005; 37:2976-2981.

7. Rivolta R, Castagnone D, Elli A, Quarto Di Palo P. Evaluation of kidney graft function by arterial flow using color Doppler flowmetry. Eur J Ultrasound 1996; 3:223-229. 
8. Kahraman S, Genctoy G, Cil B, et al. Prediction of renal allograft function with early Doppler ultrasonography. Transplant Proc 2004; 36:13481351.

9. Radermacher J, Mengel M, Ellis S, et al. The renal arterial resistance index and renal allograft survival. NEngl J Med 2003; 349:115-124.

10. Krumme B. Renal Doppler sonography: update in clinical nephrology. Nephron Clin Pract 2006; 103:c24-c28.

11. Nezami N, Tarzamni MK, Argani H, Nourifar M. Doppler ultrasonographic indexes in kidney transplant recipients: its relationship with kidney function. Iran J Kidney Dis 2007; 1:82-87.

12. Heine GH, Gerhart MK, Ulrich C, Köhler H, Girndt M. Renal Doppler resistance indices are associated with systemic atherosclerosis in kidney transplant recipients. Kidney Int 2005; 68:878-885.

13. Heine GH, Girndt M, Sester U, Köhler H. No rise in renal Doppler resistance indices at peak serum levels of cyclosporin A in stable kidney transplant patients. Nephrol Dial Transplant 2003; 18:1639-1643.

14. Krumme B, Grotz W, Kirste G, Schollmeyer P, Rump LC. Determinants of intrarenal Doppler indices in stable renal allografts. J Am Soc Nephrol 1997; 8:813-816.

15. Chiang YJ, Chu SH, Chuang CK, et al. Resistive index cannot predict transplant kidney function. Transplant Proc 2003; 35:94-95.

16. Jimenez C, Lopez MO, GonzalezE, Selgas R. Ultrasonography in kidney transplantation: values and new developments. Transplant Rev (Orlando) 2009; 23:209-213.

17. Cosgrove DO, Chan KE. Renal transplants: what ultrasound can and cannot do. Ultrasound Q2008; 24:77-87.

18. Solez K, Colvin RB, Racusen LC, et al. Banff07 classification of renal allograft pathology: updates and future directions. Am J Transplant 2008; 8:753-760.

19. Martinoli C, Bertolotto M, Crespi G, Pretolesi F, Valle M, Derchi LE. Duplex Doppler analysis of interlobular arteries in transplanted kidneys. Eur Radiol 1998; 8:765-769.

20. Martinoli C, Crespi G, Bertolotto $M$, et al. Interlobular vasculature in renal transplants: a power Doppler US study with MR correlation. Radiology 1996; 200:111-117.

21. Tublin ME, Bude RO, PlattJF. The resistive index in renal Doppler sonography: where do we stand? AJR Am J Roentgenol 2003; 180:885-892.

22. Weitzel WF, Kim K, Rubin JM, Xie H, O'Donnell M. Renal advances in ultrasound elasticity imaging: measuring the compliance of arteries and kidneys in end-stage renal disease. Blood Purif 2005; 23:10-17.

23. Tublin ME, Tessler FN, Murphy ME. Correlation between renal vascular resistance, pulse pressure, and the resistive index in isolated perfused rabbit kidneys. Radiology 1999; 213:258-264.

24. MurphyME, Tublin ME. Understanding the Doppler RI: impact of renal arterial distensibility on the RI in a hydronephrotic ex vivo rabbit kidney model. J Ultrasound Med 2000; 19:303-314.

25. Bude RO, Rubin JM. Relationship between the resistive index and vascular compliance and resistance. Radiology 1999; 211:411-417.

26. Bude RO, Rubin JM. Effect of downstream cross-sectional area of an arterial bed on the resistive index and the early systolic acceleration. Radiology 1999; 212:732-738.
27. Luo Y,LiMX,LI KP,Luo ZJ. The study of the relationship between renal hemodynamic changes and asphyxia degree in neonatal asphyxia [in Chinese]. JUltrasound Clin Med 2009; 11:825-827.

28. Bohlouli A, Tarzamni MK, Zomorrodi A, Addollahifard S, Hashemi B, Nezami N. Postnephrectomy changes in Doppler indexes of remnant kidney in unrelated kidney donors. Urol J2009; 6:194-198.

29. Nakai A, Asakura H, Oya A, Yokota A, Koshino T, Araki T. Pulsed Doppler US findings of renal interlobar arteries in pregnancy-induced hypertension. Radiology 1999; 213:423-428.

30. MacIsaac RJ, Thomas MC, Panagiotopoulos S, et al. Association between intrarenal arterial resistance and diastolic dysfunction in type 2 diabetes. Cardiovasc Diabetol 2008; 7:15.

31. Parolini CP, Noce A, Staffolani E, Giarrizzo GF, Costanzi S, Splendiani G. Renal resistive index and long-term outcome in chronic nephropathies. Radiology 2009; 252:888-896.

32. ChudekJ, Kolonko A, Król R, Ziaja J, Cierpka L, WiecekA. The intrarenal vascular resistance parameters measured by duplex Doppler ultrasound shortly after kidney transplantation in patients with immediate, slow, and delayed graft function. Transplant Proc 2006; 38:42-45.

33. Galesić K, Sabljar-Matovinović M, Tomić M, Brkljacić B. Renal vascular resistance in glomerular disease-correlation of resistance index with biopsy findings. Coll Antropol 2004; 28:667-674. 